\title{
OUTPUT PREDICTION UNDER RANDOM MEASUREMENTS. AN LMI APPROACH
}

\author{
I. Peñarrocha ${ }^{*, 2}$ A. Sala ${ }^{* *}$ R. Sanchis * \\ P. Albertos ${ }^{* *}$ \\ * Departament de Tecnologia, Universitat Jaume I \\ Campus de Riu Sec, 12071 Castelló, Spain. \\ e-mail: ipenarro@tec.uji.es, rsanchis@tec.uji.es \\ ** Departamento de Ingeniería de Sistemas y Automática \\ Universidad Politécnica de Valencia \\ Apdo. 22012, E-46071, Valencia, Spain. \\ e-mail: asala@isa.upv.es, pedro@aii.upv.es
}

\begin{abstract}
In this paper, the design of an output predictor in a system with random scarce sampling is addressed. A model based predictor that takes into account the past measured outputs is used, and a Lyapunov function of the estimation error is used for design purposes. The Lyapunov design problem becomes a feasibility problem over a set of linear matrix inequalities applying the Schur complement formula. Three different design approaches have been developed. Some examples show the performances of each approach. Copyright (c)2005 IFAC
\end{abstract}

Keywords: missing-data, random sampling, unconventional sampling, time-varying sampling periods, martingale convergence, linear matrix inequalities.

\section{INTRODUCTION}

In many industrial applications the control signal is updated at a fixed rate $T$, but the output is measured with a different timing pattern and, sometimes, by various sensors, each one having a maybe different sampling rate, and reliability. In some practical cases the output is not available at every sampling time due to computer overload, communication errors, shared sensors or event-driven sensors. Different authors have dealt with the modeling of such systems when the measurement pattern is periodic (Salt et al.

\footnotetext{
1 This project has been partially granted by the CICYT project number DPI2002-04432.

2 Supported through FPI grant number CTBPRB/2002/245 from Conselleria de Cultura, Educació $i$ Esport from Generalitat Valenciana
}

(1993), Araki and Hagiwara (1986), Araki (9), Khargonekar et al. (1985), Kranc (1957)), based on the definition of a model that relates outputs measured at one rate with inputs updated at another rate. This allows, for example, to tackle the problem of the design of a dual-rate control system Albertos et al. (1996). However, none of them try to explicitly estimate the outputs at the instants when they are unavailable, in order to apply standard control.

In works like, Albertos and Goodwin (2002) and Sanchis and Albertos (2002) an internal model of the system has been used to estimate the missing outputs with the use of a Kalman filter with high complexity and computational cost, and in Albertos et al. (1999) an external model was used leading to a lower complexity and computational 
cost. As an alternative to the Kalman filter for discrete-time linear systems, several authors have addressed the filtering problem as a convex optimization problem using linear matrix inequalities techniques Palhares and Peres (2000); Wang et al. (2004, 1999); Xie et al. (2004); Xu and Chen (2003). Nevertheless, none of them considered the case of scarce measurements as Kalman filter does.

This paper deals with the synthesis of a predictor that estimates the unmeasured outputs at period $T$ from the random measured data and using an input-output model of the system.

LMI quadratic decay rate conditions will be set up to obtain proper predictor gains. The result of the synthesis process allows the designer to specify the desired minimum decay rate and the number of intersampling periods.

The layout of this paper is as follows: In section 2 the sampling scenario and the control scheme are described, including the predictor algorithm. In section 3 the equation that defines the output estimation error dynamics is derived, and the design guidelines are shown for three different techniques. Some numerical examples are analyzed on section 4 , showing how the use of an adequate predictor improves the output estimation for a given sampling scenario. Finally on section 5 the main conclusions are summarized.

\section{PROBLEM STATEMENT}

\subsection{Control scheme}

Consider the digital control system shown in figure 2.1, where $G(s)$ is a CT SISO linear process whose input is updated at period $T$ by a computer with a zero-order hold. A new input $u[t]$ arrives every control period, where $t \in \mathbb{N}$ is the number of input update. The relationship between the discrete signal and the continuous one is

$$
u[t]=u(t T) .
$$

The process output $y(t)$ is measured synchronously with the input update at a different and no necessarily constant period. The $k$-th measured output is denoted with $y_{k}$. If the number of input updates from $y_{k-1}$ to $y_{k}$ is denoted with $N_{k}$ (figure 2.1), a relationship between the sequence of measured outputs $\left(y_{k}\right)$, the output discrete signal $(y[t])$, and the continuous one $(y(t))$ is established as

where

$$
y_{k}=y\left[t_{k}\right]=y\left(t_{k} T\right)
$$

$$
t_{k}=\sum_{j=1}^{k} N_{j}
$$

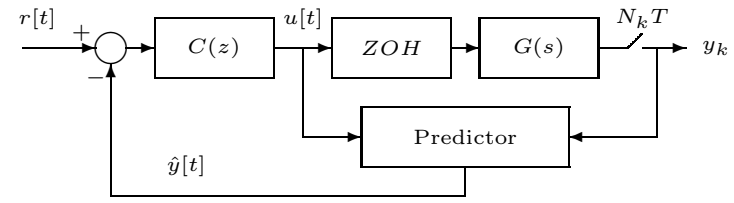

Fig. 1. Control Scheme.

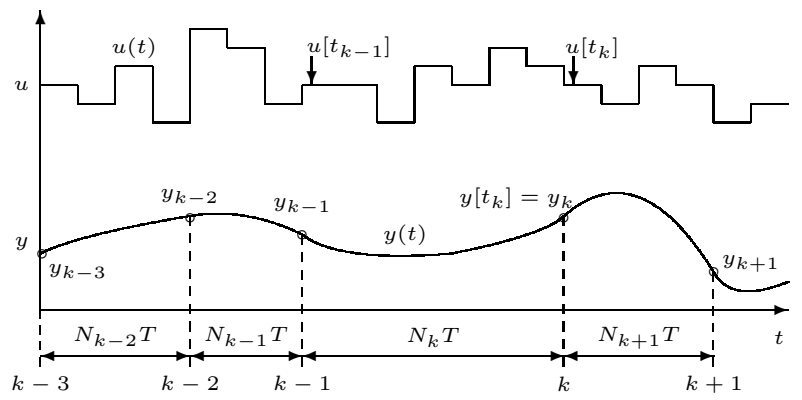

Fig. 2. Synchronous random sampling.

represents the instant in which the $t$-th input update occurs and the $k$-th output measurement is available.

In the control scheme, $C[z]$ represents the conventional linear digital controller that operates at period $T, \hat{y}[t]$ is the output sequence of the predictor that is fed back to the controller to compute the control action.

\subsection{Plant}

The discrete $\mathrm{ZOH}$ equivalent of $G(s)$ at period $T$, is defined by the difference equation

$$
y[t]=-\boldsymbol{\theta}_{a}^{\boldsymbol{\top}} Y[t-1]+\boldsymbol{\theta}_{b}^{\boldsymbol{\top}} U[t-1],
$$

where $\boldsymbol{\theta}_{a}=\left[\begin{array}{lll}a_{1} & \cdots & a_{n}\end{array}\right]^{\top}$ and $\boldsymbol{\theta}_{b}=\left[\begin{array}{lll}b_{1} & \cdots & b_{n}\end{array}\right]^{\top}$ are the parameter vectors, $Y[t-1]=[y[t-1] \cdots y[t-n]]^{\top}$ is the output vector and $U[t-1]=[u[t-1] \cdots u[t-n]]^{\top}$ is the input vector (with $y[t]=y(t T), u[t]=$ $u(t T))$. Equation (2) can be rewritten as

$$
\begin{aligned}
Y[t] & =\left[\begin{array}{c}
-\boldsymbol{\theta}_{a}^{\boldsymbol{\top}} \\
\boldsymbol{I}_{(n-1) \times(n)}
\end{array}\right] Y[t-1]+\left[\begin{array}{c}
\boldsymbol{\theta}_{b}^{\boldsymbol{\top}} \\
\mathbf{0}_{(n-1) \times(n)}
\end{array}\right] U[t-1] \\
& =\boldsymbol{A} Y[t-1]+\boldsymbol{B} U[t-1],
\end{aligned}
$$

where $\boldsymbol{I}_{(n-1) \times(n)}$ are the $n-1$ first rows and $n$ first columns of the identity matrix, and $\mathbf{0}_{(n-1) \times(n)}$ is a null matrix of order $(n-1) \times(n)$.

\subsection{Predictor}

The digital controller $C[z]$ needs the sequence of outputs at period $T$, but the process output is measured irregularly at a slower rate, therefore, the missing inter-sampling measurements have to be estimated. For that purpose, a model based output predictor has been added in the closedloop. The predictor (introduced in Albertos et al. (1999) and analyzed in depth in Sanchis (1999)) 
uses the parameters of the process model, and all the previous inputs, measurements and output estimates to obtain the prediction of the unknown outputs. When there is no measurement available, the predictor runs the model in open loop, leading to

$$
\hat{Y}[t]=\boldsymbol{A} \hat{Y}[t-1]+\boldsymbol{B} U[t-1],
$$

where $\hat{Y}[t]$ is the estimation of the output regression vector at instant $t T$. When there is a new measurement (i.e. $y[t]$ is available), the estimated output regression vector is given by

$$
\begin{aligned}
\hat{Y}[t]= & (\boldsymbol{I}-\boldsymbol{l}[t] \boldsymbol{h})(\boldsymbol{A} \hat{Y}[t-1]+\boldsymbol{B} U[t-1]) \\
& +\boldsymbol{l}[t] \boldsymbol{h} Y[t] .
\end{aligned}
$$

where $\boldsymbol{h}=\left[\begin{array}{llll}1 & 0 & \cdots & 0\end{array}\right]$, and the vector gain $\boldsymbol{l}[t]=$ $\left[l_{1}[t] \cdots l_{n}[t]\right]^{\top}$ must be designed to assure the predictor stability, as it will be shown in the following sections. If the availability factor $\delta[t]$ is defined $(\delta[t]=1$ if there is one measurement available, $\delta[t]=0$ if not), then the output estimation can be computed every control updating instant as

$$
\begin{aligned}
\hat{Y}[t]= & (\boldsymbol{I}-\boldsymbol{l}[t] \boldsymbol{h} \delta[t])(\boldsymbol{A} \hat{Y}[t-1]+\boldsymbol{B} U[t-1]) \\
& +\boldsymbol{l}[t] \boldsymbol{h} \delta[t] Y[t] \\
\hat{y}[t]= & \boldsymbol{h} \hat{Y}[t] .
\end{aligned}
$$

\section{PREDICTOR DESIGN}

\subsection{Prediction error}

Theorem 1. The prediction error dynamics of the algorithm (6) when there is no modeling error is modeled by a linear time-varying system defined at measuring instants by

$$
E_{k}=\left(\boldsymbol{I}-\boldsymbol{l}_{k} \boldsymbol{h}\right) \boldsymbol{A}^{N_{k}} E_{k-1}=\boldsymbol{M}_{k} E_{k-1},
$$

where $N_{k}$ may change arbitrarily with time, and the prediction error is only defined at measuring instants as

$$
E_{k} \equiv E\left[t_{k}\right]=Y\left[t_{k}\right]-\hat{Y}\left[t_{k}\right] \equiv Y_{k}-\hat{Y}_{k} .
$$

PROOF. At the measuring instant $t=t_{k}$, the prediction is obtained by (5), and subtracting $Y[t]$ in that expression, it is obtained that

$E[t]=(\boldsymbol{I}-\boldsymbol{l}[t] \boldsymbol{h})(Y[t]-(\boldsymbol{A} \hat{Y}[t-1]+\boldsymbol{B} U[t-1]))$.

If equations (3) and (4) are applied recursively, $Y[t]$ and $\hat{Y}[t-1]$ can be expressed as a function of the regression vectors in the previous sampling time (i.e, $Y\left[t-N_{k}\right]$ and $\hat{Y}\left[t-N_{k}\right]$ ) and the corresponding inputs between measurements, leading to

$E[t]=(\boldsymbol{I}-\boldsymbol{l}[t] \boldsymbol{h})\left(\boldsymbol{A}^{N_{k}} Y\left[t-N_{k}\right]-\boldsymbol{A}^{N_{k}} \hat{Y}\left[t-N_{k}\right]\right)$, that can be expressed as (7) taking into account that instant $t$ corresponds to the $k$-th sampling time (see figure 2.1).

\subsection{Design guidelines}

3.2.1. Nominal Stability If a periodic sampling is addressed $\left(N_{k}=N\right.$, where $N$ is a constant value) under the absence of disturbances $(w(t)=$ $0)$, a constant gain is used $\left(\boldsymbol{l}_{k}=\boldsymbol{l}\right)$ and the predictor dynamics is stable if and only if the eigenvalues of the matrix $(\boldsymbol{I}-\boldsymbol{l} \boldsymbol{h}) \boldsymbol{A}^{N}$ are inside the unit circle. This can be achieved, for instance, applying pole placement.

3.2.2. Robust Stability When a random sampling is addressed ( $N_{k}$ varies randomly with time), the previous condition for all $k$ is not necessary neither sufficient, and the only possibility to prove the stability is the use of Lyapunov functions. Linear matrix inequalities will be used to find a stable predictor for a set of models at different sampling rates.

Three possibilities are studied here. First, a predictor that uses a constant vector $\boldsymbol{l}$ is used, assuring either a monotonous decrease of the Lyapunov function of the prediction error, or, when this is not possible, an asymptotical convergence in average. In both cases the samples do not need to be time tagged. Finally, the design is also addressed with the use of a variable vector $\boldsymbol{l}_{k}$, only applicable with time tagged samples.

3.2.3. Constant gain The simplest algorithm that can be outlined is derived for the case where a constant vector $\boldsymbol{l}$ is used for the prediction law to assure the convergence of the prediction error.

Monotonous decrease of the Lyapunov function for the prediction error.

Theorem 2. The prediction error of the algorithm defined by (6) when there is one measurement available every $\Delta$ input updating periods, being $\Delta$ a discrete independent random variable with positive integer values, is stable with a minimum decay rate of $\mu \in(0,1]$ if the set of LMIs

$$
\left[\begin{array}{cc}
\mu^{2} \boldsymbol{P} & \left((\boldsymbol{P}-\boldsymbol{X} \boldsymbol{h}) \boldsymbol{A}^{\Delta_{j}}\right)^{\top} \\
(\boldsymbol{P}-\boldsymbol{X} \boldsymbol{h}) \boldsymbol{A}^{\Delta_{j}} & \boldsymbol{P}
\end{array}\right]>\mathbf{0}
$$

are verified for all $\Delta_{j}$ in an expected range of variation $\mathcal{N}=\left\{\Delta_{1}, \ldots, \Delta_{m}\right\} . \boldsymbol{P}$ and $\boldsymbol{X}$ are the matrix variables of the LMI problem, and the predictor gain is $\boldsymbol{l}=\boldsymbol{P}^{-1} \boldsymbol{X}$. 
PROOF. Defining the Lyapunov function $V_{k}=$ $E_{k}^{\top} \boldsymbol{P} E_{k}$, and using equation (7) with a constant gain $\boldsymbol{l}$ it is easy to show that

$$
V_{k}=E_{k-1}^{\top} \boldsymbol{M}_{k}^{\top} \boldsymbol{P} \boldsymbol{M}_{k} E_{k-1},
$$

with $\boldsymbol{M}_{k}=(\boldsymbol{I}-\boldsymbol{l} \boldsymbol{h}) \boldsymbol{A}^{N_{k}}$. A sufficient condition to assure stability with a decay rate $\mu$ (in the sense $\left.V_{k}<\mu^{2} V_{k-1}\right)$, is that there exists one matrix $\boldsymbol{P}>\mathbf{0}$ such that

$$
\begin{gathered}
\boldsymbol{M}\left(\Delta_{j}\right)^{\top} \boldsymbol{P} \boldsymbol{M}\left(\Delta_{j}\right)-\mu^{2} \boldsymbol{P}<\mathbf{0}, \\
j=1, \ldots, m
\end{gathered}
$$

where

$$
\boldsymbol{M}\left(\Delta_{j}\right)=(\boldsymbol{I}-\boldsymbol{l} \boldsymbol{h}) \boldsymbol{A}^{\Delta_{j}} .
$$

This set of inequations can be joined in the LMI problem (8) using the Schur complement formula Boyd et al. (1994). Expressing equation (9) as

$$
\begin{gathered}
\mu^{2} \boldsymbol{P}-\boldsymbol{M}\left(\Delta_{j}\right)^{\top} \boldsymbol{P} \boldsymbol{P}^{-1} \boldsymbol{P} \boldsymbol{M}\left(\Delta_{j}\right)>\mathbf{0} \\
j=1, \ldots, m
\end{gathered}
$$

and taking into account restriction $\boldsymbol{P}>\mathbf{0}$, both inequalities can be joined together in the expression

$$
\left[\begin{array}{cc}
\mu^{2} \boldsymbol{P} & \boldsymbol{M}\left(\Delta_{j}\right)^{\top} \boldsymbol{P} \\
\boldsymbol{P} \boldsymbol{M}\left(\Delta_{j}\right) & \boldsymbol{P}
\end{array}\right]>\mathbf{0} ; \quad j=1, \ldots, m
$$

which leads to

$$
\begin{array}{cc}
\mu^{2} \boldsymbol{P} & \left((\boldsymbol{I}-\boldsymbol{l} \boldsymbol{h}) \boldsymbol{A}^{\Delta_{j}}\right)^{\top} \boldsymbol{P} \\
\boldsymbol{P}(\boldsymbol{I}-\boldsymbol{l} \boldsymbol{h}) \boldsymbol{A}^{\Delta_{j}} & \boldsymbol{P} \\
j=1, \ldots, m,
\end{array}
$$

if expression (10) is used. Defining $\boldsymbol{X}=\boldsymbol{P} \boldsymbol{l}$ as a new matrix variable, the linear matrix inequality (8) is obtained.

Asymptotical convergence in average. When the previous procedure does not give any feasible solution, the problem can be addressed assuring that the error converges to zero in average, as it is shown in the following result. Convergence in average might not preclude, however, the possibility of undesirable short transients under some (worstcase) low-likelihood sampling patterns.

Theorem 3. Under the same hypothesis of the previous theorem, the prediction error of the algorithm defined by (6) converges asymptotically to 0 with probability 1 and with a discrete decay rate faster than $\mu \in(0,1]$ if there exists one matrix $\boldsymbol{P}$ symmetric and positive definite such that

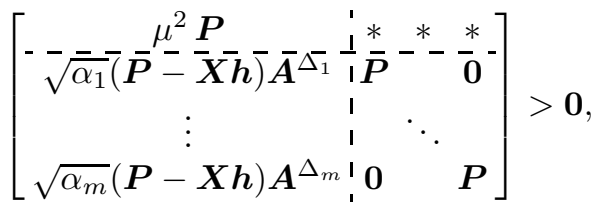

where $\alpha_{j}$ is the probability assigned to every possible intersampling period $\Delta_{j} \in \mathcal{N}$ and $* * *$ denotes the necessary terms to build a symmetric matrix. $\boldsymbol{P}$ and $\boldsymbol{X}$ are again the matrix variables, and the predictor gain is $\boldsymbol{l}=\boldsymbol{P}^{-1} \boldsymbol{X}$.

PROOF. The proof is based on theorem 8 (in the annex). From the Lyapunov function $V_{k}=$ $E_{k}^{\top} \boldsymbol{P} E_{k}$ and equation (7) it is easy to show that

$$
\begin{aligned}
\mathbf{E}\left\{V_{k} \mid F_{k-1}\right\} & =E_{k-1}^{\top} \mathbf{E}\left\{\boldsymbol{M}_{k}^{\top} \boldsymbol{P} \boldsymbol{M}_{k}\right\} E_{k-1} \\
& =\mu^{2} V_{k-1}-\gamma_{k},
\end{aligned}
$$

where $F_{k-1}$ is any filtration of the past information until instant $k-1, \mathbf{E}$ means mathematical expectation, $\mu^{2}$ is the minimum expected value of the discrete decay rate for the Lyapunov function, and the sequence $\gamma_{k}$ is defined as

$$
\gamma_{k}=E_{k-1}^{\top}\left(\mu^{2} \boldsymbol{P}-\mathbf{E}\left\{\boldsymbol{M}_{k}^{\top} \boldsymbol{P} \boldsymbol{M}_{k}\right\}\right) E_{k-1} .
$$

If there exists one matrix $\boldsymbol{P}>\mathbf{0}$ such that

$$
\mathbf{E}\left\{\boldsymbol{M}_{k}^{\top} \boldsymbol{P} \boldsymbol{M}_{k}\right\}-\mu^{2} \boldsymbol{P}<\mathbf{0}
$$

is fulfilled, the sequence $\gamma_{k}$ is non negative. Expressing (12) as

$$
\mathbf{E}\left\{V_{k} \mid F_{k-1}\right\}=V_{k-1}-\left(\gamma_{k}+\left(1-\mu^{2}\right) V_{k-1}\right),
$$

theorem 8 can be applied leading to $\lim _{k \rightarrow \infty} V_{k}=$ $V_{\infty}$ with probability one and $\sum_{j=1}^{\infty} \gamma_{j}<\infty$ w.p.1, that also implies that $\lim _{k \rightarrow \infty} \gamma_{k}=0$ w.p.1, and hence $\lim _{k \rightarrow \infty} E_{k}=0$ w.p.1.

Using the values of the probability assigned to each possible value $\Delta_{j}$ (i.e., $\alpha_{j}$ ), condition (13) can be written as

$$
\sum_{j=1}^{m} \alpha_{j} \boldsymbol{M}\left(\Delta_{j}\right)^{\top} \boldsymbol{P} \boldsymbol{M}\left(\Delta_{j}\right)-\mu^{2} \boldsymbol{P}<\mathbf{0},
$$

with $\boldsymbol{M}\left(\Delta_{j}\right)=(\boldsymbol{I}-\boldsymbol{l} \boldsymbol{h}) \boldsymbol{A}^{\Delta_{j}}$. The LMI (11) is obtained from (14) and condition $\boldsymbol{P}>\mathbf{0}$ via the Schur complement formula, as explained before.

\subsubsection{Sample-time dependent gain scheduling}

When the number of intersampling periods $\left(N_{k}\right)$ are available at the sampling instant $k$, a different strategy can b e used. Every time a new output is available, a gain $\boldsymbol{l}_{k}$ is used depending on $N_{k}$ to assure the stability of the predictor over a set of possible indexes $\mathcal{N}=\left\{\Delta_{1}, \ldots, \Delta_{m}\right\}$. The approach is applicable if the number of possible intersampling periods is a finite set. Then, the set of predictor gain vectors can be calculated off-line leading to the set $\{\boldsymbol{l}\}=\left\{\boldsymbol{l}\left(\Delta_{1}\right), \ldots, \boldsymbol{l}\left(\Delta_{m}\right)\right\}$.

Theorem 4. Under the same hypothesis of previous theorems, and assuming that the value $N_{k}$ is known with every measurement (see figure 2.1), a sufficient condition to assure the stability of predictor (6) with decay rate faster than $\mu \in(0,1]$ is that the set of LMIs 


$$
\left[\begin{array}{cc}
\mu^{2} \boldsymbol{P} & \left(\left(\boldsymbol{P}-\boldsymbol{X}_{\boldsymbol{j}} \boldsymbol{h}\right) \boldsymbol{A}^{\Delta_{j}}\right)^{\top} \\
\left(\boldsymbol{P}-\boldsymbol{X}_{\boldsymbol{j}} \boldsymbol{h}\right) \boldsymbol{A}^{\Delta_{j}} & \boldsymbol{P}
\end{array}\right]>\mathbf{0}
$$

is fulfilled for any $\Delta_{j} \in \mathcal{N}$, being $\boldsymbol{P}$ and $\boldsymbol{X}_{j}(j=$ $1, \ldots, m)$ the matrix variables. The predictor gain vectors depending on $\Delta_{j}$ are given by $\boldsymbol{l}\left(\Delta_{j}\right)=$ $\boldsymbol{P}^{-1} \boldsymbol{X}_{j}$.

PROOF. The proof is equivalent to the one used in theorem 2, but taking into account that now $\boldsymbol{M}_{k}=\left(\boldsymbol{I}-\boldsymbol{l}_{k} \boldsymbol{h}\right) \boldsymbol{A}^{N_{k}}$ due to the use of a sample-time dependent gain vector $\boldsymbol{l}_{k}$. A sufficient condition to assure stability with a decay rate $\mu$, is that there exists one matrix $\boldsymbol{P}>\mathbf{0}$ such that

$$
\boldsymbol{M}\left(\Delta_{j}\right)^{\boldsymbol{T}} \boldsymbol{P} \boldsymbol{M}\left(\Delta_{j}\right)-\mu^{2} \boldsymbol{P}<\mathbf{0}, \quad j=1, \ldots, m .
$$

where $\boldsymbol{M}\left(\Delta_{j}\right)=\left(\boldsymbol{I}-\boldsymbol{l}\left(\Delta_{j}\right) \boldsymbol{h}\right) \boldsymbol{A}^{\Delta_{j}}$. This set of inequations can be joined in the LMI problem (15) using Schur complements.

\section{EXAMPLES}

Example 5. Consider the continuous-time LTI system $G(s)=10 /\left(s^{3}+6 s^{2}+11 s+6\right)$ with the input being updated at a constant period $T=0.05$ seconds. Consider that the output can be measured every $\Delta$ intersampling periods, with possible values between 3 and $30(\Delta \in \mathcal{N}=$ $\{3, \ldots, 30\}, m=28)$ ). The probability assigned to each intersampling period to appear is the same (i.e., $\alpha_{i}=1 / m=0.0357$ ). If the predictor is designed to achieve a monotonous decrease of the error (1st method), only a maximum decay rate of $\mu_{1}=0.55$ can be guaranteed applying theorem 2, and the gain $\boldsymbol{l}_{1}=\left[\begin{array}{lll}1 & 0.9254 & 0.8397\end{array}\right]^{\top}$ is obtained. If the probability is also used in the design to assure the convergence in average (2nd method), a decay rate of $\mu_{2}=0.24$ can be guaranteed (applying theorem 3), obtaining the gain $\boldsymbol{l}_{2}=\left[\begin{array}{lll}1 & 0.9868 & 0.9676\end{array}\right]^{\top}$. If a sampletime dependent gain scheduling (3rd method) is designed, a decay rate faster than $\mu_{3}=0.15$ can be guaranteed (theorem 4), leading to a set of vector of gains that is not written here for brevity.

The three predictors have been simulated leading to the norm of the output error shown in figure 3 . It can be appreciated that the first method leads to a higher error than the second one that also uses a constant gain but assures the decrement of the Lyapunov function in average. The third method leads to a norm of the error similar to the one obtained with the constant gain in method 2 .

In the next example the improvement of the different predictors is analysed in a different way,

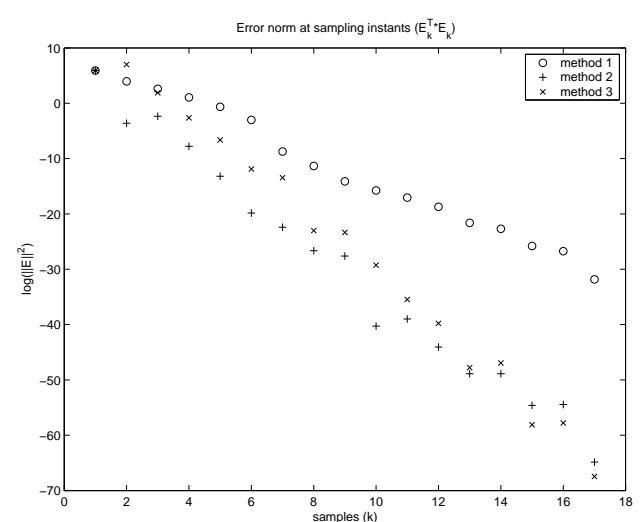

Fig. 3. Output estimation error with the three different methodologies: constant gain for monotonous decrease (o); constant gain for convergence in average $(+)$; sample-time dependent gain vector for monotonous decrease $(\times)$.

showing how the number of possible intersampling periods that leads to a feasible LMI problem changes with each design methodology.

Example 6. Consider now the unstable continuoustime LTI system $G(s)=(10 s-2) /\left(s^{2}-5 s\right)$ with the input being updated at constant period $T=0.02$ seconds. If a constant gain is applied to assure a monotonous decrease of the Lyapunov function with a decay rate faster than $\mu=0.6$ (1st method), the wider range of intersampling periods that leads to a feasible solution of the associated LMI problem is $\mathcal{N}_{1}=\{1,2,3\}$, leading to the gain $\boldsymbol{l}=[1.00220 .5341]$. If the set includes more intersampling periods, the problem (8) is not feasible, and a convergence faster than $\mu=0.6$ is not assured.

If the probability assigned to every sampling instant is assumed to be the same, i.e., $\alpha_{i}=1 / m$ $(i=1, \ldots, m, m=\operatorname{length}(\mathcal{N}))$ the convergence in average of the predictor can be assured for all the intersampling periods included in the set $\mathcal{N}_{2}=\{1, \ldots, 17\} \quad(m=17$ applying the 2 nd method). Here has been shown how the design with the second method is not as restrictive as the design with the first method. If a time-varying gain is used (3rd method), the upper limit can be extended up to 30 samples, at least, as found by the LMI settings and, as both $A^{N_{k}}$ and the gain $l_{k}$ seem to converge for long inter-sampling times, possibly to an infinite number of samples.

\section{CONCLUSIONS}

In this work, the design of an output predictor for systems under random scarce measurements has been addressed. The predictor uses the inputoutput model of the system and the previous 
inputs and measured outputs to predict the output when there is no measurement available. The number of input updates between measurements is assumed to be a random value that belongs to a known finite set.

A Lyapunov function of the prediction error at the measuring instants has been defined to analyze the convergence. The Lyapunov stability problem has been transformed into a feasibility problem over a set of linear matrix inequalities by means of the Schur complement formula. The resulting LMI problem is then solved (if feasible) to calculate the predictor gain vector.

Three different design approaches have been compared using this technique: on the first one, a constant gain vector is used in order to achieve a monotonous decrease of the Lyapunov function. On the second one, a constant gain vector is used in order to achieve a convergence in average of the Lyapunov function, taking into account the probability associated to each possible number of intersampling periods. On the third one, a set of vector gains is used in order to achieve a monotonous decrease of the Lyapunov function. Each vector gain is associated to one of the possible values of the intersampling periods that must be known via time tagged samples.

The inclusion of the disturbance, noise measurement and modeling error effect on the predictor design is being developed for future works.

\section{REFERENCES}

P. Albertos and G.C. Goodwin. Virtual sensors for control applications. Annual Reviews in Control, 26:101-112, 2002.

P. Albertos, J. Salt, and J. Tornero. Dual rate adaptive control. Automatica, 31(7):1017-1030, 1996.

P. Albertos, R. Sanchis, and A. Sala. Output prediction under scarce data operation: Control applications. Automatica, 35:1671-1681, 1999.

M. Araki. Recent development in digital control theory. Proc. 12th IFAC World Congr., 951-960 (1993), 9.

M. Araki and T. Hagiwara. Pole assignment by multirate data output feedback. International Journal of Control, 44(6):1661-1673, 1986.

S. Boyd, L. Gaoui, E. Feron, and V. Balakrishnan. Linear Matrix Inequalities in Systems and Control Theory. SIAM. Philadelphia, 1994.

P.P. Khargonekar, K. Poolla, and A. Tannenbaum. Robust control of linear time-invariant plants using periodic compensation. IEEE Trans. Automatic Control, 30:1088-1096, 1985.

G.M. Kranc. Input-output analysis of multirate feedback systems. IRE Trans. Auto. Control, 31(2):315-319, 1957.
R.M. Palhares and P.L.D. Peres. Robust filtering with guaranteed energy-to-peak performance an lmi approach. Automatica, 36:851-858, 2000.

M.M. Rao. Foundations of stochastic Analysis. Academic Press, 1981.

J. Salt, P. Albertos, and J. Tornero. Modelling of non-conventional sampled data systems. 3rd IEEE Control Applications Conference, 2:631635, 1993.

R. Sanchis. Control of Industrial Processes with Scarce Measurements. Doctoral Thesis, Universidad Politécnica de Valencia, Spain., 1999.

R. Sanchis and P. Albertos. Recursive identification under scarce measurements. convergence analysis. Automatica, 38:535-544, 2002.

J. Wang, C. Wang, and H. Gao. Robust $\mathcal{H}_{\infty}$ filtering for lpv discrete-time state-delayed systems. Nature and Science, 2(2):36-45, 2004.

Z. Wang, B. Huang, and H. Unbehauen. Robust $\mathcal{H}_{\infty}$ observer design of linear state delayed systems with parametric uncertainty: the discretetime case. Automatica, 35:1161-1167, 1999.

L. Xie, L. Lu, D. Zhang, and H. Zhang. Improved robust $\mathcal{H}_{2}$ and $\mathcal{H}_{\infty}$ filtering for uncertain discrete-time systems. Automatica, 40:873880, 2004.

S. $\mathrm{Xu}$ and $\mathrm{T}$. Chen. Robust $\mathcal{H}_{\infty}$ filtering for uncertain impulsive stochastic systems under sampled measurements. Automatica, 39:509516, 2003.

\section{Appendix A. MARTINGALES CONVERGENCE THEOREM}

First, asymptotic convergence with probability 1 is defined for a stochastic process.

Definition 7. A temporal serie $e_{k}$ is said to converge asymptotically to 0 with probability 1 , if $\forall \epsilon>0, \delta>0, \exists m>0$ such that $P\left(e_{k}>\epsilon\right)<$ $\delta \quad \forall k>m$.

The next theorem about martingales convergence can be found in Rao (1981) as theorem 7, and it says:

Theorem 8. (Rao (1981)). Let $V_{k}, \alpha_{k}$ and $\beta_{k}$ be sequences non negatives of random variables. If

$$
\mathbf{E}\left(V_{k} \mid F_{k-1}\right) \leq V_{k-1}+\alpha_{k}-\beta_{k}
$$

and

$$
\sum_{j=1}^{\infty} \alpha_{j}<\infty \text { with probability one, }
$$

then $V_{k} \stackrel{k \rightarrow \infty}{\longrightarrow} V_{\infty}$ with probability one, and $\sum_{j=1}^{\infty} \beta_{j}<\infty$ with probability one. 